\title{
Single-pulse femtosecond laser fabrication of concave microlens- and micromirror arrays in chalcohalide glass
}

\author{
Viktor Kadan ${ }^{\mathrm{a}}$, Ivan Blonskyi ${ }^{\mathrm{a}}$, Yevhen Shynkarenko ${ }^{\mathrm{a}}$, Andriy Rybak ${ }^{\mathrm{a}}$, \\ Laurent Calvez ${ }^{\mathrm{b}}$, Bohdan Mytsyk ${ }^{\mathrm{c}}$, Oleh Shpotyuk ${ }^{\mathrm{d}, \mathrm{e}}$ \\ ${ }^{a}$ Institute of Physics of the NAS of Ukraine, Prospect Nauky 46, 03680 Kyiv, Ukraine; \\ ${ }^{b}$ UMR-CNRS 6226, Université de Rennes 1, 35042 Rennes Cedex, France; \\ ${ }^{c}$ Karpenko Physico-Mechanical Institute of the NAS of Ukraine, Naukova str. 5, 79060 Lviv, \\ Ukraine; \\ ${ }^{d}$ Vlokh Institute of Physical Optics, Dragomanov str. 23, 79005 Lviv, Ukraine; \\ ${ }^{e}$ Institute of Physics of Jan Dlugosz University, al. Armii Krajowej 13/15, 42200 Czestochowa, \\ Poland
}

The diffraction-limited plano-concave microlens- and micromirror arrays were produced in chalcohalide glass of $65 \mathrm{GeS}_{2}-25 \mathrm{Ga}_{2} \mathrm{~S}_{3}-10 \mathrm{CsCl}$ composition transparent from $\sim 0.5$ to $11 \mu \mathrm{m}$. Only a single 200 fs laser pulse with $800 \mathrm{~nm}$ central wavelength is required to form microlens, which after metal coating becomes a concave micromirror. This process can serve as a basis for flexible technology to fabricate regular microlens and micromirror arrays for optotelecom applications, its performance being limited only by repetition rate of the laser pulses (typically 1000 microlenses per second).

Keywords: Chalcohalide glass; femtosecond laser; microlens array; micromirror; laser ablation

\section{Introduction}

The need of telecommunications and microelectronics in integration and miniaturization of functional elements has led to emergence of micro-optics, i.e. small optical elements by the control of light pulses, in particular, gratings, polarizers, mirrors and microlenses, that can be used for radiation coupling and out-coupling into- and from waveguides, micro-resonators, twodimensional microlens arrays for light detector matrices, etc. [1,2]. In many special applications, the glassy-like functional media have a lot of advantages among other alternative candidates in view of their high efficiency, rich diversity of different light-induced modification phenomena suitable for device production, and unprecedented exploitation reliability [3,4].

Chalcogenide compounds are known to compose an important materials platform for promising micro-optics exploring wide spectral range from visible to mid-IR and far-IR covering both commercially important atmospheric telecommunication windows at 3-5 and 8-12 $\mu \mathrm{m}$ [5]. In structurally-disordered state, these compounds are typically presented by chalcogenide glasses (ChG), e.g. vitreous alloys of chemical elements from IV-V-th groups of the Periodic table (such as $\mathrm{As}, \mathrm{Ge}, \mathrm{Sb}, \mathrm{Bi}$, etc.) with chalcogens ( $\mathrm{S}, \mathrm{Se}, \mathrm{Te})$ prepared by melt-quenching, which can be recognized as one of most technologically promising media for numerous applications in civil areas, including fiber IR sources, laser technique with power delivery systems, optical amplifiers, switches, scanning near field microscopy, chemical sensing, imaging, measurements, 
control, etc. [5-7]. In some active photonics using, the $\mathrm{ChG}$ are often modified with Ga additions (preferentially in the form of sulfides such as $\mathrm{Ga}_{2} \mathrm{~S}_{3}$ ) allowing their further doping with rareearth elements to ensure for example second harmonic generation in mid-IR [8]. Optical transmittance in visible range of these glasses can be partly controlled due to metal halides (such as $\mathrm{CsCl}, \mathrm{KBr}$, CsI, etc.), thus forming a large family of perspective optoelectronics media termed as chalcogenide-halide (chalcohalide) glasses (ChHG) [8-10].

As to micro-optics modification technologies, the recent progress has been achieved mainly in application to spatially-homogeneous glassy-like chalcogenides (the undoped ChG). Thus, the fabrication technology of micro-optical elements using ChG photoresist was developed in [1114]. Particularly in [14], inexpensive and reliable fabrication of ultrathin Fresnel lenses using ChG photoresists was demonstrated. The photoinduced modifications of structure and refractive index of ChG occurring during fs laser irradiation and their applications for writing waveguiding structures have been reviewed in [15], while in [16], the main attention was paid to reversible nonlinear changes of refractive index under the fs laser irradiation.

Convex microlenses were produced from ChG using printing method [17] and photoexpansion phenomenon [18-21]. Concave microlenses were fabricated exploring the phenomenon of gigantic photocontraction [22]. In [23,24], the concave microlens arrays were produced in $\mathrm{ChG}$ combining fs laser irradiation followed by acid etching. Microlens arrays were fabricated by modifying microstructure of lithium aluminosilicate Foturan glass using fs laser direct writing followed by thermal treatment, wet etching, and annealing [25].

Unfortunately, both photoexpansion and photocontraction in ChG are subject to longterm relaxation, thus deteriorating temporal stability of the obtained microoptical elements. Moreover, production of a single lens takes a comparatively long time in above processes. Both productivity and long-term stability can be improved using fs lasers, which have significant advantages over traditional laser sources. In fact, combination of high light field intensity and ultrashort pulse duration provides fast and deterministic deposition of the pulse energy into transparent material. As a result, the material removal occurs before the heat damage, thus enabling high-quality micromachining with nanometric resolution (see, e.g. [26,27]).

Nonetheless, the ablated surfaces of high optical quality are difficult to produce even with fs laser pulses because of ripple formation [28,29]. It was shown that despite conventional wisdom on non-thermal nature of ablation with fs laser pulses, thin residual melted layer remains below the ablated region in borosilicate glass [30-32], and silicon [33]. The force of surface tension flattens the liquid layer, making the surface of ablation craters comparatively smooth after solidification. Thus, fabrication of microlens with a single pulse of fs laser looks attractive from a productivity standpoint, under the condition that a satisfactory surface quality is provided. 
To the best of our knowledge, only one group reported direct fabrication of microlens arrays in organic compounds using single-pulse fs laser radiation for each lens, these being concave arrays in polydimethylsiloxane (PDMS) [34] and convex lens arrays in polymethylmethacrylate (PMMA) [35]. Since PDMS and PMMA are IR-opaque, a highly productive single-step technology of microlens fabrication in IR-transparent materials is still required.

In this paper, we describe a rapid process of direct single-pulse fabrication of microlenses developed for $\mathrm{ChHG}$. Since various fabrication techniques of embedded waveguides in $\mathrm{ChG}$ media have previously been developed [36,37], this process opens the way to fabricate complex integrated 3D micro-optics.

\section{Experimental results and discussion}

The specimens of ChHG composed of $65 \% \mathrm{GeS}_{2}, 25 \% \mathrm{Ga}_{2} \mathrm{~S}_{3}$, and $10 \% \mathrm{CsCl}$ have been obtained from high purity raw materials ( $\mathrm{Ge}, \mathrm{Ga}, \mathrm{S}$ and $\mathrm{CsCl}$ of $5 \mathrm{~N}$ purity) using conventional melt-quenching route as was described in details elsewhere [9,10,38]. The purified ingredients weighed in stoichiometric proportions were inserted in a silica ampoule under vacuum $\left(10^{-4} \mathrm{~Pa}\right)$. The sealed ampoule was placed in a rocking furnace during several hours at $850^{\circ} \mathrm{C}$ and quenched in water at room temperature. The polished $(\lambda / 4)$ cubic sample $(5 \times 5 \times 5 \mathrm{~mm})$ was cut from the resulting material, this sample being finally annealed $10^{\circ} \mathrm{C}$ below the glass transition temperature ( $\mathrm{Tg}$ of $405^{\circ} \mathrm{C}$ ) for $4 \mathrm{~h}$ to reduce residual mechanical stress induced during the quench. This glass composition presents a large window of transparency ranging from $\sim 0.5 \mu \mathrm{m}$ to $11 \mu \mathrm{m}[10]$.

Single-pulse ablation experiments were performed using the setup shown in Fig. 1.

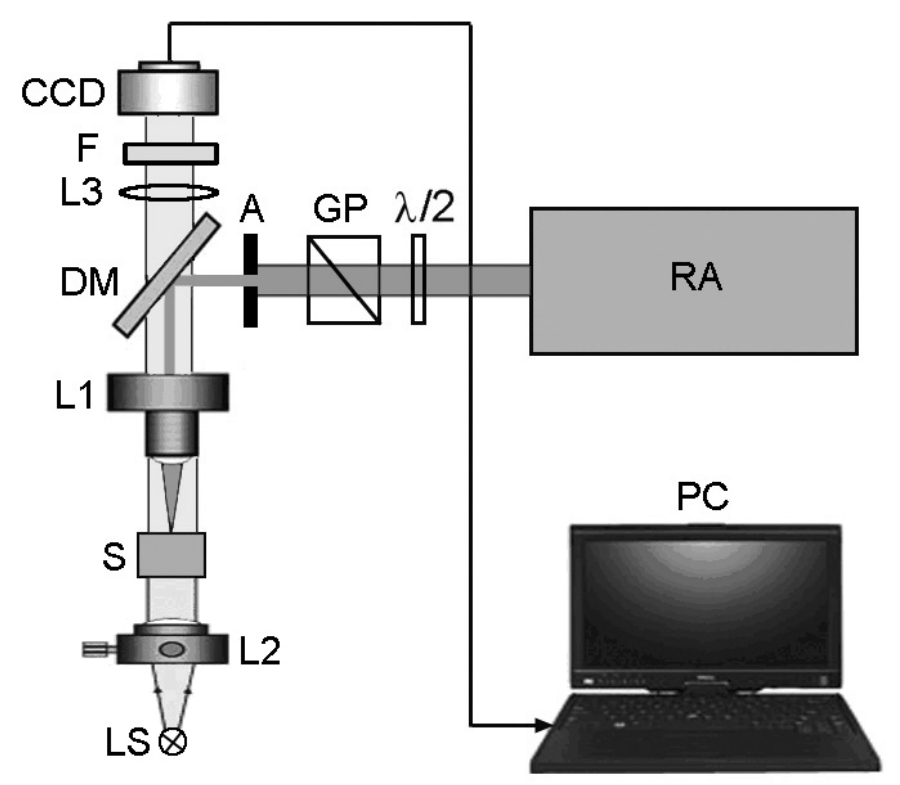

Fig. 1. Schematic view of the experimental set-up for single-pulse ablation. 
The regenerative amplifier RA (Legend F-1K-HE) produces a train of horizontally polarized laser pulses $(2.5 \mathrm{~mJ}$ maximum pulse energy, $200 \mathrm{fs}$ pulse width, $800 \mathrm{~nm}$ central wavelength). The polarization plane can be rotated with a half-wave plate $\lambda / 2$, thus varying the energy of the pulse which passes a vertically arranged Glan polarizer GP. Having passed the aperture A with the diameter varying from 1 to $3 \mathrm{~mm}$ and reflected from the dichroic mirror DM, the beam enters the microscope objective lens L1 $(3.7 \times, 0.11$ NA). A centrally peaked Airy intensity pattern is formed in the focal plane of L1 lens close to the entrance surface of the sample $\mathrm{S}$ as a result of diffraction on the aperture A [39]. Typical intensity distribution of laser pulse, recorded in the focal plane of L1 lens at $2 \mathrm{~mm}$ diameter of aperture A, is shown in Fig. 2a.

Laser ablation was carried out with single laser pulses of different energy varying from 5 to $25 \mu \mathrm{J}$ at different positions $\delta( \pm 400 \mu \mathrm{m})$ of the focal plane of the lens L1 relative to the surface of the sample S. The processing area is imaged on the CCD matrix with lenses L1 and L3 in transmitted light of the incandescent lamp source LS, which is collimated with the lens L2. Glass color filter F cuts off the back-reflected laser light at $800 \mathrm{~nm}$.

Optical and SEM microscopic study of the sample after single-shot laser exposure show ablation craters with predominantly smooth surface (Fig. 2b,c,d). In view of very small linear absorption in $\mathrm{GeS}_{2}-\mathrm{Ga}_{2} \mathrm{~S}_{3}-\mathrm{CsCl} \mathrm{ChHG}$ at $800 \mathrm{~nm}$ [40], only nonlinear absorption provides the surface energy deposition from laser pulse needed for ablation. Apart from the surface ablation, the question of the laser-induced alterations of the bulk material, which could deteriorate optical performance of the formed microelements, is also important. We carefully examined the material of the ChHG sample beneath the surface of the ablated area for the presence of damage traces or modification of refractive index, but no inhomogeneities have been revealed under the microscopic inspection through the polished lateral face of the sample. However, as will be shown below, the energy of the laser pulse, remaining after the surface absorption, is sufficient to produce fs filaments, which concentrate high energy density in its core. Presumably, they can cause the material modification or/and damage. It is this approach we used earlier to write filament-induced waveguides in glassy $\mathrm{As}_{4} \mathrm{Ge}_{30} \mathrm{~S}_{66}$ [36]. However, the writing process in [36] was cumulative, taking more than $20 \mathrm{~s}$ exposure time at $1 \mathrm{kHz}$ pulse repetition rate to produce appreciable change of refractive index, while a single laser pulse caused no modification of the material. Thus, we conclude that only surface ablation modifies the sample in the present singlepulse process, while possible filamentation leaves in the sample no bulk damage, which could deteriorate the optical performance of the resulting microoptical elements in transmission mode. This conclusion, as shown below, is confirmed by the ability of the produced microlenses to form focal spots and images of sufficient quality. 
It is known, that in transparent dielectrics the surface laser breakdown threshold for nanosecond laser pulses is much lower than the bulk one, due to the influence of surface defects or surface-assisted air breakdown [41], but for fs laser pulses the question is more complicated [42]. D. Von der Linde et al. [43] explain the observed decrease of thresholds of fs breakdown on the surface by effect of contamination or surface imperfections. In our case, occurrence of the surface breakdown is most likely due to strong two-photon absorption (TPA). Indeed, the band gap of the studied ChHG $\left(E_{\mathrm{g}} \sim 3 \mathrm{eV}\right)$ roughly equals to twice laser photon energy $h v=1.55 \mathrm{eV}$, thus causing strong TPA and two-step absorption by laser-induced plasma, which attenuate the pulse in the surface layer. Assuming Airy pattern as the intensity distribution of the laser beam focused on the sample surface [44], we obtain that for a typical pulse energy of $E_{\mathrm{p}}=12 \mu \mathrm{J}$ and duration of $\tau_{\mathrm{p}}=200 \mathrm{fs}$, the peak power density in the center of the laser spot of $32.4 \mu \mathrm{m}$ diameter (Fig. 2a) $I_{0}=2.7 \cdot 10^{13} \mathrm{~W} / \mathrm{cm}^{2}$. Using the TPA coefficient for $\mathrm{As}_{4} \mathrm{Ge}_{30} \mathrm{~S}_{66} \beta=8 \times 10^{-10} \mathrm{~cm} / \mathrm{W}[45]$ as an estimate, and assuming the TPA law as

$$
I(x)=\frac{I_{0}}{1+\beta x I_{0}},
$$

where $x$ is the length of beam path inside the material, we find that the initial intensity is halved on the depth of $0.5 \mu \mathrm{m}$ due to TPA. This explains the surface character of the ablation even without taking into account plasma absorption.

The ablation craters have different diameters, depending on the pulse energy and the distance $\delta$ of the focal plane of the lens L1 from the surface of the sample. The optimal pulse energy $E_{\mathrm{p}}(5-15 \mu \mathrm{J})$ and $\delta( \pm 100 \mu \mathrm{m})$ ranges have been found, which resulted in the ablation zone without considerable deterioration of the surface quality and with sharp boundaries, which indicate presence of the ablation threshold. Considering the radial intensity distribution in the Airy disc, which is described by the square of the Bessel function of the first kind $J_{1}$ [39]:

$$
I(\theta)=I_{0}\left(\frac{2 J_{1}(k a \sin \theta)}{k a \sin \theta}\right)^{2},
$$

where $I_{0}$ is the maximum intensity in center of the Airy disc; $a$ is the aperture radius, $k=2 \pi / \lambda$ is the wavenumber and $\theta$ is the observation angle, we have estimated the single-pulse ablation intensity threshold from the crater size at $E_{\mathrm{p}}$ ranging from 5 to $25 \mu \mathrm{J}$ as $2.5 \pm 0.5 \cdot 10^{12} \mathrm{~W} / \mathrm{cm}^{2}$.

The area modified with single laser pulse (12 $\mu \mathrm{J}$ energy) at $\delta=0$ is shown on Fig. 2b,c,d. 

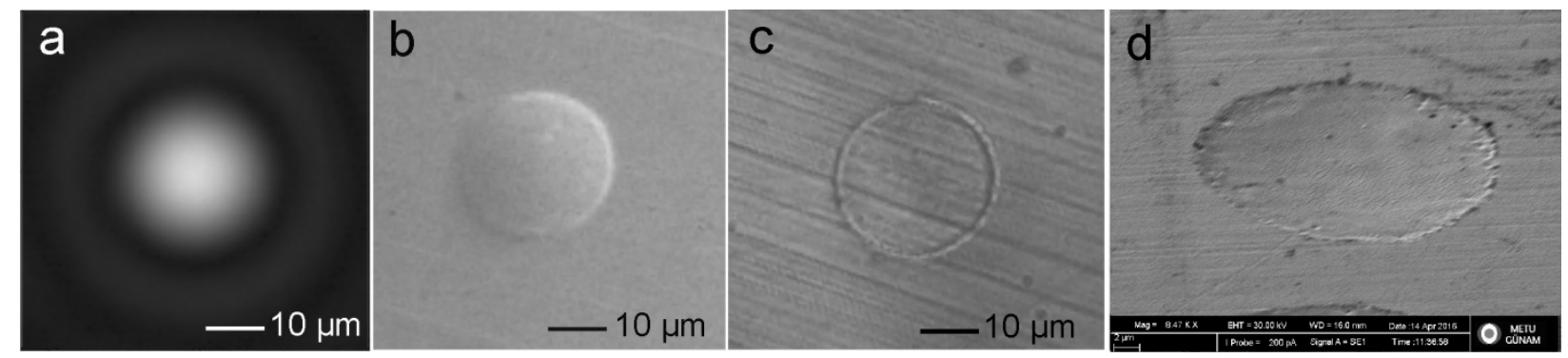

Fig. 2. Airy pattern of $32.4 \mu \mathrm{m}$ diameter formed in the focal plane of $3.7 \times$ lens at $2 \mathrm{~mm}$ diameter of the aperture A1 (a). The ablated area, after the exposure to a single laser pulse of $12 \mu \mathrm{J}$ energy at $\delta=0$ in lateral light (b), in back-reflection (c); taken on SEM (d)

Note, that optical quality of the modified surface in the ablated spot is almost unaltered in comparison with the original surface of the sample, as seen from Fig.2b,c,d. No permanent change of the surface reflectivity is observed after the ablation (Fig. 2c). Average gray level integrated over the ablated area in Fig. 2b,c is the same, as in its nearest surroundings, indicating that light scattering by the ablated surface is not increased after the single-pulse exposure. However, already the second laser pulse deteriorates the previously single-pulse-exposed surface. The smooth surfaces of the craters produced by fs laser have been observed earlier in borosilicate glass [30-32] and in PDMS [34]. It was found, that, despite the conventional wisdom that fs laser pulses cause direct ablation skipping the liquid phase, residual melted layer remains after the ejection of major amount of material, which is smoothed by the high-pressure blast wave and the forces of surface tension before solidification. In order to find out whether the same physical mechanism underlies the formation of the smooth surface, we investigate the stages of the laser-ChHG interaction with time-resolved microscopy (Fig. 3a).
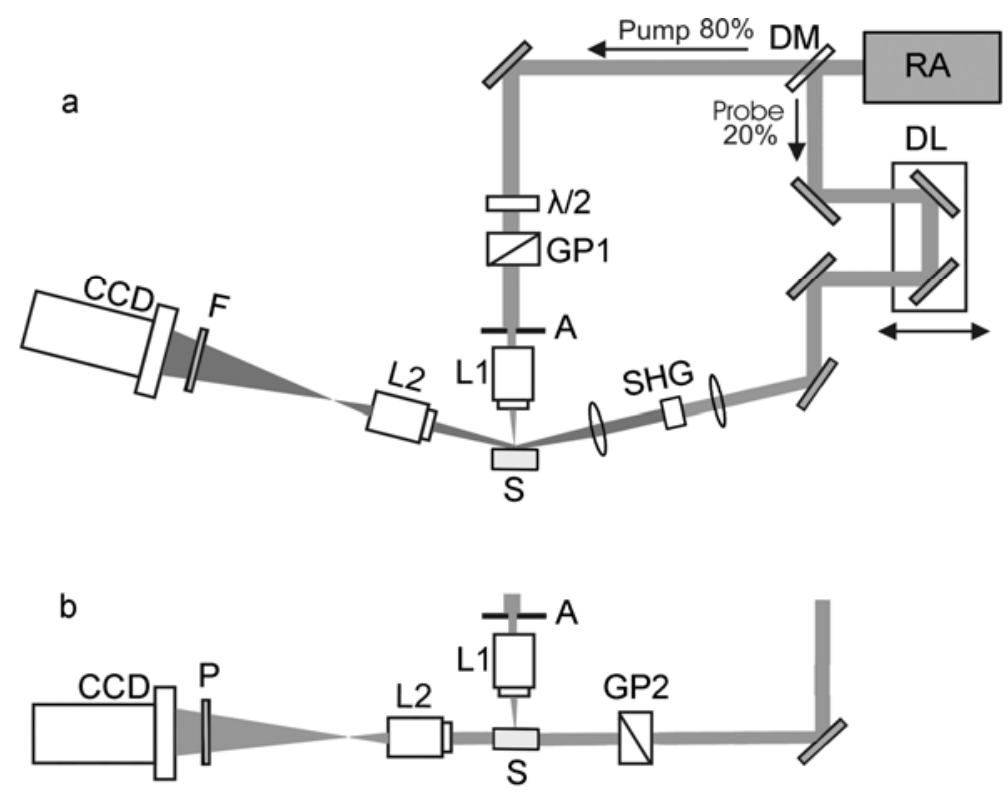
Fig. 3. Time-resolved microscopy setups: the combined transient transmission/reflection microscopy (a); the transient polarization microscopy (b).

The excitation is carried out normally to the sample $\mathrm{S}$ surface in the same way, as in the setup, shown in Fig. 1, but the excitation spot and the surrounding area is probed with the frequency-doubled laser pulse, which is temporally delayed up to $8 \mathrm{~ns}$ with respect to the pump pulse with the delay line DL. The DL provides variable temporal delays up to $1.8 \mathrm{~ns}$. To obtain longer delays, we fixed additional mirrors in the probe optical path. The probe beam is directed at an angle $13^{\circ}$ to the sample surface and reflected into the long-working-distance objective L2 $(16 \times, 0.2 \mathrm{NA})$. Note that unlike previous studies of fs laser ablation dynamics [46-49], such pump-probe geometry allows observing simultaneously both reflection from the sample surface and transmission above the excitation spot. The objective L2 forms the image on the CCD matrix. Cut-off glass filter F protects the matrix from the fundamental wavelength component of the probe and the scattered pump laser light. After every laser shot the sample is shifted to newly expose an undamaged area. To improve the picture quality, the reference snapshot of the undamaged surface, taken before ablation, was subtracted from ablation snapshot, adding then the average gray level.

The upper fragment in Fig. 4 shows the time-integrated picture of the glowing jet of ablation plasma at $E_{\mathrm{p}}=15 \mu \mathrm{J}$. In addition to the proper plasma jet, its reflection from the sample surface can be seen on right from the ablation spot. The pictures below show several transient stages of the ablation at different time delays $\tau_{\mathrm{d}}$ indicated in their upper left side. Note, that $\tau_{\mathrm{d}}$ is a relative value, because the exact time difference between arrival of the pump and probe pulses cannot be determined. We designated as 0 the delay corresponding to the first detectable darkening of the excited spot, which is probably caused by optical properties of superheated melted material [49] and scattering of the probe light at the early stages of ablation process. Apart from the increasing darkening at $0<\tau_{\mathrm{d}}<27 \mathrm{ps}$, noticeable transversal expansion of the ablation spot starts with delay of $127 \mathrm{ps,} \mathrm{indicating} \mathrm{the} \mathrm{onset} \mathrm{of} \mathrm{material} \mathrm{ejection} \mathrm{from} \mathrm{the}$ excited area. In addition to the material ejection, we observe formation of the blast wave of expanding plasma detaching from the ablation spot, which contributes to the surface smoothening. The first signs of the blast wave formation appear starting from $\tau_{\mathrm{d}}=1.7 \mathrm{~ns}$. The blast wave front becomes visible due to the abrupt change in the refraction index at the plasma/air interface. The front has an aspherical shape, which indicates anisotropic expansion of the ablation species, its maximum velocity, as calculated from the last two pictures at 4.3 and $7.6 \mathrm{~ns}$ delay, is $9.7 \mathrm{~km} / \mathrm{s}$, i.e. much higher than the sound velocity in the air. The presence of the toroidal formation seen on the crater edges at $1.7-7.6$ ns delays may be indicative of transversal 
expulsion of the molten layer driven by blast wave pressure. Note that no marked rim remains at the crater edge after solidification (Fig. 2d).
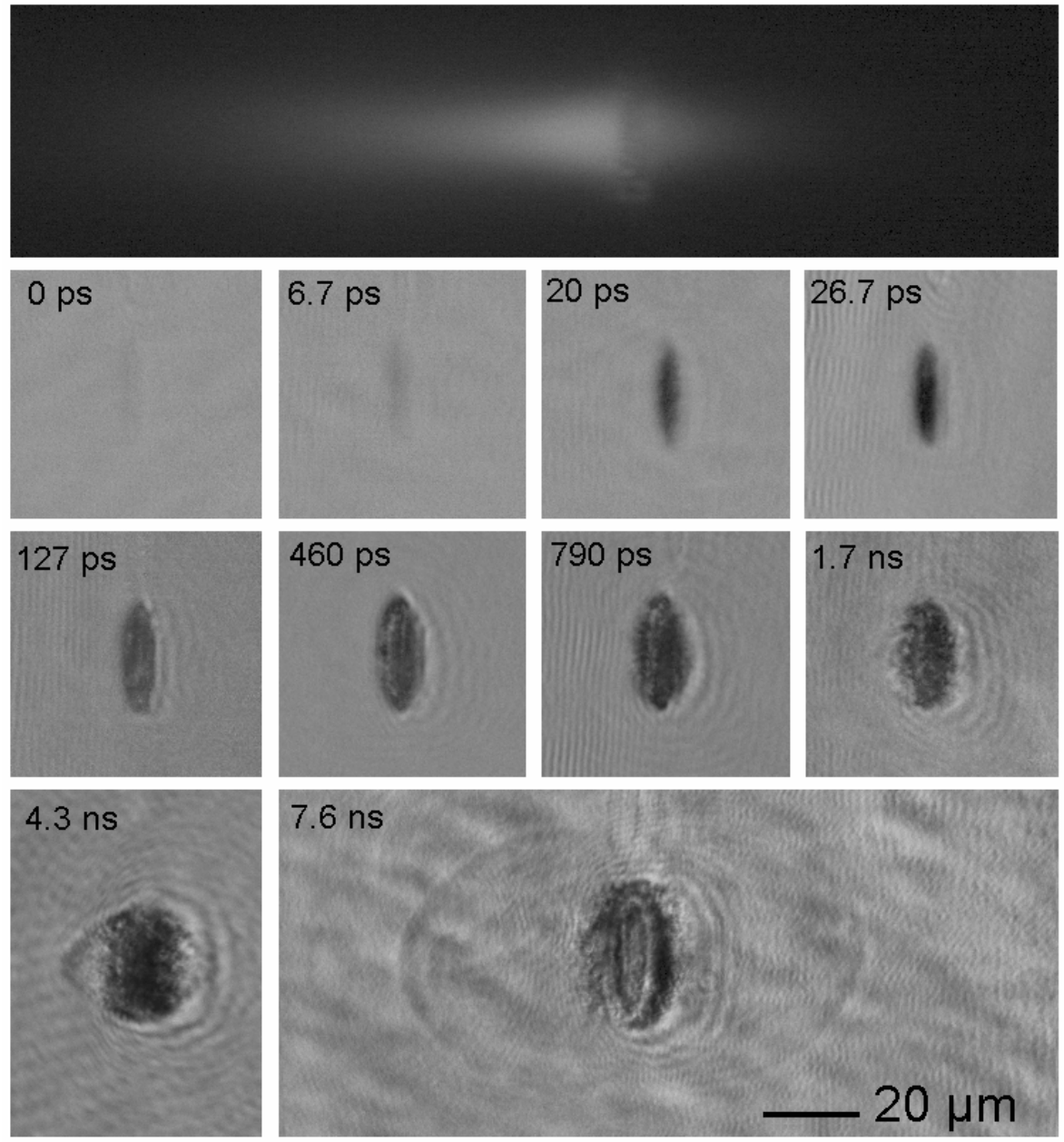

Fig. 4. Time-integrated picture of the glowing ablated plasma at $E_{\mathrm{p}}=15 \mu \mathrm{J}$ (uppermost) and snapshots of the ablation process at different time delays. The scale is the same for each picture.

So, the results of the present experiment confirm the appearance of the blast wave in the process of single-shot ablation of $\mathrm{ChHG}$ of $65 \mathrm{GeS}_{2}-25 \mathrm{Ga}_{2} \mathrm{~S}_{3}-10 \mathrm{CsCl}$ composition, which contributes to the smoothening of the crater surface, as proposed in [30-32].

However, a considerable part of the pulse, which remains after the surface absorption, propagates inside the material, being subjected to self-focusing and filamentation. Considering the importance of these effects in fs machining of functional glassy media [36,45], we studied fs pulse propagation inside the ChHG with time-resolved polarization microscopy setup [51,52 5153]. Fig. $3 \mathrm{~b}$ shows only a part of the whole setup, which differs from that, shown in Fig. $3 \mathrm{a}$. Vertically polarized laser pulse of much lower energy $E_{\mathrm{p}}=100 \mathrm{~nJ}$ is focused on the sample surface. No observable surface damage occurs at this energy. Temporally delayed pulse of the same wavelength, polarized at $45^{\circ}$ with GP2 Glan prism probes the excitation pulse path inside the sample. If the axis of the polarizer P is set perpendicular to that of GP2, no light normally 
reaches the CCD matrix. The excitation pulse induces local anisotropic change of the original refractive index in the sample owing to electronic Kerr effect. The induced anisotropy zone, which displays the spatial intensity distribution of the pulse, moves together with the pulse due to the small response time (about $1 \mathrm{fs}$ ) of electronic Kerr effect - the dominant mechanism of the nonlinear index change [51-53]. As a result, it induces a small ellipticity in the originally planepolarized probe pulse. The orthogonal component of the polarization survives the polarizer P, thus forming an instant image (or polarogram) of the moving pulse, which represents the intensity distribution in the pulse at the moment of arrival of the probe pulse.

Fig. 5 shows the shape evolution of the femtosecond laser pulse travelling inside the $65 \mathrm{GeS}_{2}-25 \mathrm{Ga}_{2} \mathrm{~S}_{3}-10 \mathrm{CsCl}$ glass at different probe delays. It is seen, that the pulse self-contracts transversally to the diameter of $\sim 7 \mu \mathrm{m}$ in the $2.0 \mathrm{ps}-4.67 \mathrm{ps}$ delay range. This value is much smaller than the diameter of the diffraction-limited focal spot of $32.4 \mu \mathrm{m}$ in the linear propagation mode, thus indicating self-focusing and filamentation process in the ChHG. Note, that $E_{\mathrm{p}}=100 \mathrm{~nJ}$ slightly exceeds the threshold energy of filamentation, which we estimated as $\sim 70 \mathrm{~nJ}$ in this material. In addition, the snapshots in Fig. 5 make it possible to find the pulse propagation velocity, and eventually, the refractive index of the material $(n=2,16)$, in close agreement with $\mathrm{n}=2.12$ at $633 \mathrm{~nm}[10]$.

Summarizing, the femtosecond laser pulse supplies the energy needed for surface ablation due to TPA. Material ejection from the crater, apart from evaporation, is also provided by high-pressure supersonic blast wave. The residual thin layer of liquid, solidifying, acquires optically smooth surface under the action of forces of surface tension. The remaining part of the initial pulse most likely propagates in filamented or multifilamented mode inside the sample, causing, however, no further material alteration or damage in the single-pulse regime.
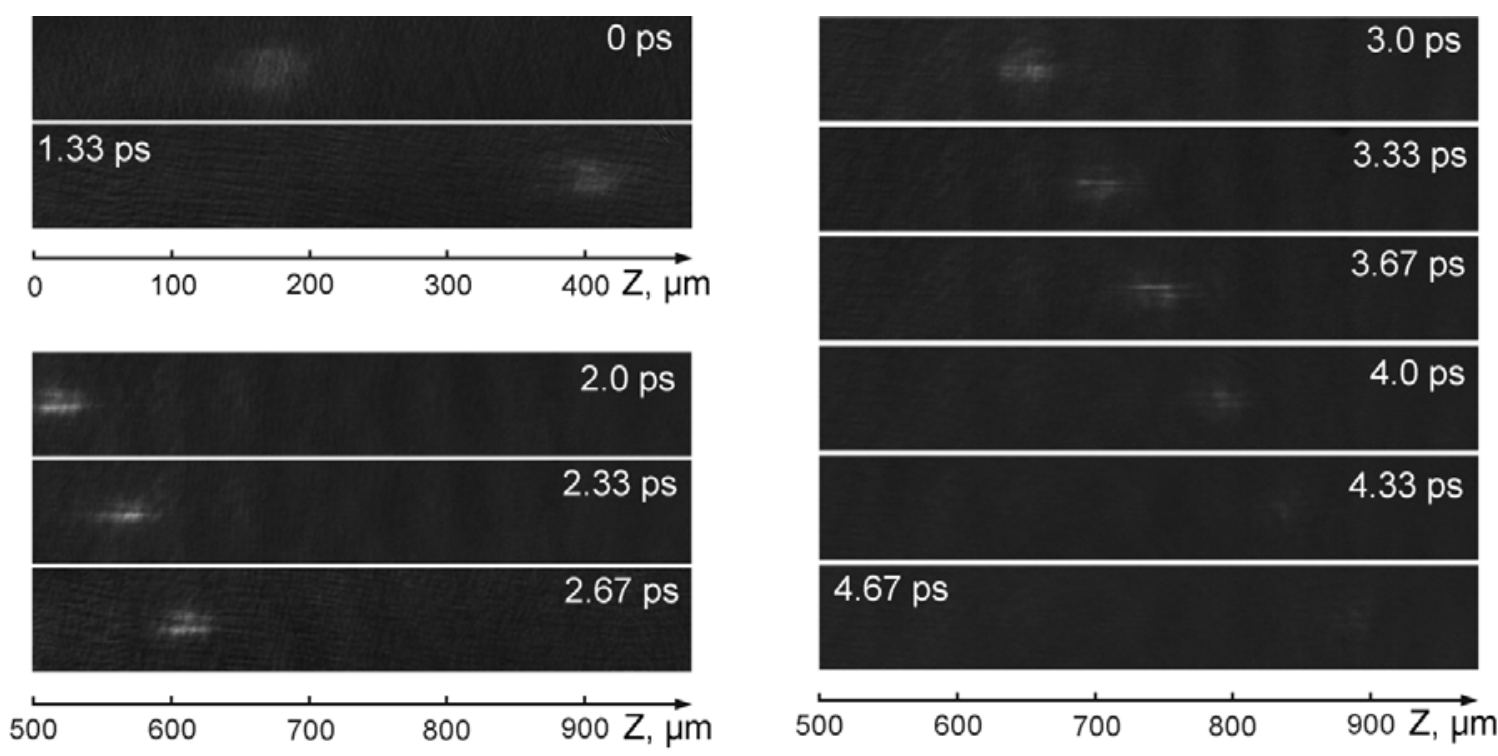

Fig. 5. Polarization microscopy pictures of femtosecond laser pulse with $E_{p}=100 \mathrm{~nJ}$ 
at different time delays, propagating in $\mathrm{ChG}$ of $65 \mathrm{GeS}_{2}-25 \mathrm{Ga}_{2} \mathrm{~S}_{3}-10 \mathrm{CsCl}$ type.

The vertical and horizontal scales are the same.

Microinterferometric picture of the single-pulse crater at $E_{\mathrm{p}}=12 \mu \mathrm{J}$ taken under the incandescent lamp illumination is shown in Fig. 6a. The direction of the deflection of the interference fringes indicates the deepening on the sample surface. Basing on trigonometric calculations of the shape of the fringes, omitted here for brevity, we have fitted the crater surface with a sphere of radius $R=159 \mu \mathrm{m}$. Under assumption that $n=2.16$, as shown above, the focal distance $f$ of the produced microlens according to the thin-lens formula $f=R /(n-1)$ is $137 \mu \mathrm{m}$. This value agrees well with the experimentally measured virtual focal distance $140 \mu \mathrm{m}$ of the lens. The central depth of the microlens crater is $0.5 \mu \mathrm{m}$. The light intensity distribution in transmission mode of the microscope at the depth of $140 \mu \mathrm{m}$ beneath the surface is shown in Fig. 2b. It is characterized by a bright virtual focal spot of diffraction-limited diameter $7.7 \mu \mathrm{m}$ between the zeros, according to the formula $d=2.44 \lambda f / D$, where $D$ is the microlens diameter. The virtual image of letters IP (Fig. 6c) formed by microlens characterizes its quality. The letters have been printed on the paper and illuminated with white light as shown in Fig. 6d.

Varying the pulse energy and focusing conditions, we produced the microlenses of 15 to $35 \mu \mathrm{m}$ diameter and 50 to $200 \mu \mathrm{m}$ focal distance.
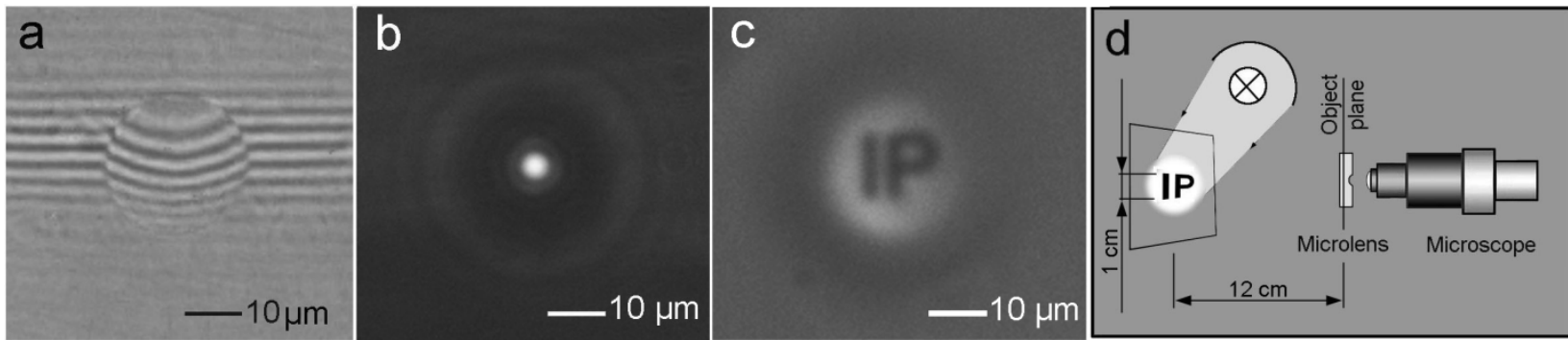

Fig. 6. Microinterferogram of the microlens formed on the surface of the sample $\mathrm{S}$ after the single-pulse exposure $\left(E_{\mathrm{p}}=12 \mu \mathrm{J}, \delta=0\right)$ (a). The virtual focal spot of the microlens at the depth of $140 \mu \mathrm{m}$ beneath the surface of the sample under the LED illumination at $\lambda=0.63 \mu \mathrm{m}(\mathrm{b})$. The virtual image of the letters "IP" formed by the microlens (c). The measurement geometry (d).

Scanning the sample surface with focused laser beam in pulse repetition mode opens the way to rapid and flexible technology of fabrication of microlens arrays. We covered the $1 \times 1 \mathrm{~mm}$ surface plot of the ChHG sample by concave microlenses, each of them produced by a single laser pulse at $100 \mathrm{~Hz}$ repetition rate. Fig. 7 shows the array of microlenses of $17 \mu \mathrm{m}$ diameter 
and $100 \mu \mathrm{m}$ focal distance each, which forms the arrays of virtual foci and virtual images of the letters "IP". Synchronization of the laser pulses with the stage movement is still needed to achieve a regular pattern of the array.
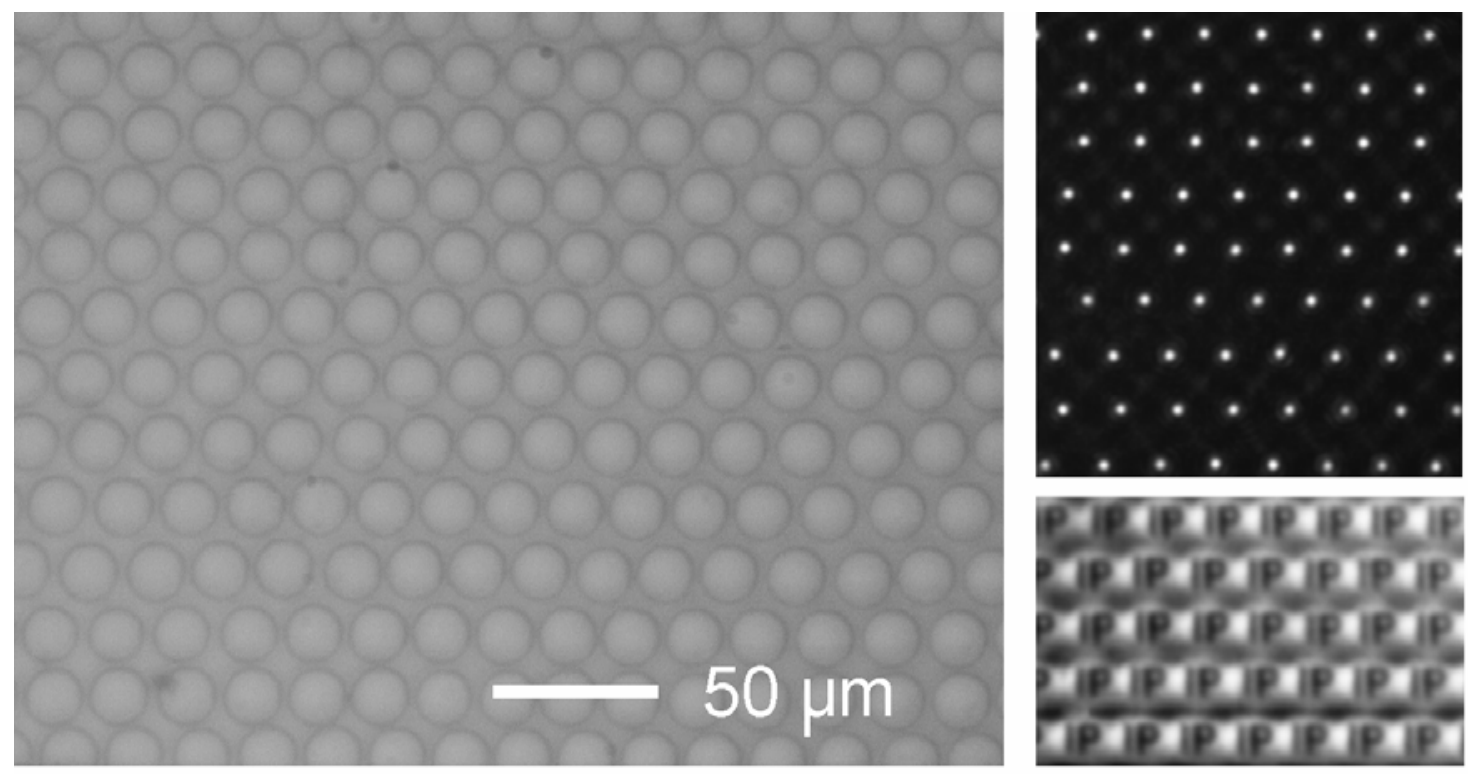

Fig. 7. Array of microlenses, virtual focal spots and virtual images of the letters "IP".

While preparing the microlens array for SEM microscopy we had coated the sample surface with a $20 \mathrm{~nm}$-thick gold layer, thus creating concave spherical micromirrors with focal distance twice as short as that of microlenses. Such micromirrors and their arrays, which in distinct from microlenses, form real focal spots and images, can find possible applications in photonics as microcavities or light concentrators for detector arrays.

\section{Conclusions}

In conclusion, we demonstrate a rapid and flexible process of production of ChHG-based diffraction-limited microlenses and micromirrors. Each lens is produced by a single fs laser pulse, which is absorbed in the surface layer due to TPA. Apart from superheating, high-pressure blast wave assists the material ejection from the ablation crater. The residual thin layer of liquid, solidifying, acquires optically smooth surface under the action of forces of surface tension. The part of the initial pulse which remains after TPA, most likely propagates in filamented or multifilamented mode inside the sample, causing, however, no further material alteration or damage in the single-pulse regime. Array of microlenses was produced by scanning the sample surface with focused laser beam in pulse repetition mode. Metal coating transforms the microlens array into an array of micromirrors. Arbitrarily complex array geometry can easily be achieved just by programming the scanning sequence. Rapid production technology of microlens- and micromirror arrays can be developed on the basis of this method, its productivity 
being limited by the pulse repetition rate of femtosecond laser (typically $1000 \mathrm{~Hz}$ ). The ChHGbased arrays of concave microlenses can be used as molds for replication of polymer-based convex microlens arrays.

\section{Acknowledgements}

This research was performed using experimental equipment of the Laser Femtosecond Center for Collaborative Use of the National Academy of Sciences of Ukraine. We acknowledge support from the NAS of Ukraine and the Science and Technology Center in Ukraine (project 6174), as well as from the NAS of Ukraine and the Scientific and Technological Research Council of Turkey (a joint research project), and to the Ukrainian State Fund for Fundamental Research (Project F73/23805).

The authors are grateful to Dr. Tahir Çolakoğlu from the Center for Solar Energy Research and Applications, Middle East Technical University, Department of Physics, Ankara, Turkey for his valuable assistance with SEM microscopy.

\section{References}

[1] S. Sinzinger, J. Jahns, Microoptics, WILEY-VCH GmbH \& Co. KGaA, Weinheim, 2003.

[2] P. Ruffieux, T. Scharf, I. Philipoussis, H. P. Herzig, R. Voelkel, K. J. Weible, Two step process for the fabrication of diffraction limited concave microlens arrays, Opt. Express 16 (2008) 19541-19549.

[3] M. Brinkman, U. Fotheringham, J.S. Hayden, Y. Okano, Glass modification techniques for photonic devices, Proc. SPIE 5061 (2003) 96-102.

[4] D.N. Krol, Femtosecond laser modification of glass, J. Non-Cryst. Solids 354 (2008) 416424.

[5] J.-L. Adam, X. Zhang, Chalcogenide glasses: Preparation, properties and applications, Woodhead Publ. Ser. in Electron. and Opt. Mat., Philadelphia-New Delhi, 2013.

[6] X. Zhang, B. Bureau, P. Lucas, C. Boussard-Pledel, J. Lucas, Glasses for seeing beyond visible, Chem Eur. J. 14 (2008) 432-442.

[7] J.-L. Adam, L. Calvez, J. Troles, V. Nazabal, Chalcogenide glasses for infrared photonics, Intern. J. Appl. Glass Sci. 6 (2015) 287-294.

[8] L. Calvez, C. Lin, M. Roze, Y. Ledemi, E. Guillevic, B. Bureau, M. Allix, X. Zhang, Similar behaviors of sulfide and selenide-based chalcogenide glasses to form glass-ceramics, Proc. SPIE 7598 (2010) 759802-1-16.

[9] P. Masselin, D. Le Coq, L. Calvez, E. Petracovschi, E. Lepine, E. Bychkov, X. Zhang, CsCl effect on the optical properties of the $80 \mathrm{GeS}_{2}-20 \mathrm{Ga}_{2} \mathrm{~S}_{3}$ base glass, Appl. Phys. A106 (2012) 697702.

[10] Y. Ledemi, L. Calvez, m. Roze, X.H. Zhang, B. Bureau, M. Poulain, Y. Messaddeq, Totally visible transparent chloro-sulphide glasses based on $\mathrm{Ga}_{2} \mathrm{~S}_{3}-\mathrm{GeS} 2-\mathrm{CsCh}$, J. Optoelectron. Adv. Mat. 9 (2007) 3751-3755.

[11] M. Manevich, M. Klebanov, V. Lyubin, J. Varshal, J. Broder, N. P. Eisenberg, Gap microlithography for chalcogenide micro-lens array fabrication, Chalcogenide Letters 5 (2008) 61-64.

[12] Y. Kumaresan, A. Rammohan, P. K. Dwivedi, A. Sharma, Large Area IR Microlens Arrays of Chalcogenide Glass Photoresists by Grayscale Maskless Lithography, ASC Appl. Mater. Interfaces 5 (2013) 7094-7100. 
[13] N. P. Eisenberg, M. Manevich, M. Klebanov, Fabrication and testing of microlens arrays for the IR based on chalcogenide glassy resists, J. Non-Cryst. Solids 198-200 (1996) 766-768.

[14] A. Kovalskiy, M. Vlcek, C. M. Waits, M. Dubey, W. R. Heffner, H. Jain, Chalcogenide glass e-beam and photoresists for ultrathin grayscale patterning. J. Micro/Nanolith MEMS MOEMS 8 (2009) 043012(1-11).

[15] L. Petit, N. Carlie, T. Anderson, J. Choi, M.Richardson, and K. C. Richardson, Progress on photoresponse of chalcogenide glasses and films to near-infrared femtosecond laser irradiation: a review, IEEE Journal of Selected Topics in Quantum Electronics 14 (2008) 1323-1334.

[16] H. Z. Tao, C. G. Lin, H. Y. Xiao, X. J. Zhao, Z. W. Wang, S. S. Chu, S. F. Wang, Q. H. Gong, Ultrafast nonresonant third-order optical non-linearity of the 0.64GeS2-0.16Ga2S3$0.2 \mathrm{CsCl}$ chalcohalide glass. J. Mater. Sci. 41 (2006) 6481-6484.

[17] E. A. Sanchez, M. Waldmann, C. B. Arnold, Chalcogenide glass microlenses by inkjet printing, Applied Optics 50 (2011) 1974-1978.

[18] A. Velea, F. Jipa, M. Zamfirescu, R. Dabu, Femtosecond laser processing of chalcogenide glasses, Journal of Intense Pulsed Lasers and Applications in Advanced Physics 3 (2013) 27-36.

[19] H. Hisakuni, K. Tanaka, Optical fabrication of microlenses in chalcogenide glasses, Opt. Lett. 20 (1995) 958-960.

[20] S. Ramachandran, J. C. Pepper, D. J. Brady, S. G. Bishop, Micro-optical lenslets by photoexpansion in chalcogenide glasses, Journal of Lightwave Technology 15 (1997) 1371-1377.

[21] A. Saitoh, K. Tanaka, Self-developing aspherical chalcogenide-glass microlenses for semiconductor lasers, Appl. Phys. Lett. 83 (2003) 1725-1727.

[22] L. Calvez, Z. Yang, P. Lucas, Reversible giant photocontraction in chalcogenide glass, Opt. Express 17 (2009) 18581-18589.

[23] F. Chen, H. Liu, Q. Yang, X. Wang, C. Hou, H. Bian, W. Liang, J. Si, X. Hou, Maskless fabrication of concave microlens arrays on silica glasses by a femtosecond-laser-enhanced local wet etching method, Opt. Express 18 (2010) 20334-20343.

[24] A. Pan, B. Gao, T. Chen, J. Si, C. Li, F. Chen, X. Hou, Fabrication of concave spherical microlenses on silicon by femtosecond laser irradiation and mixed acid etching, Opt. Express 22 (2014) 15245-15250.

[25] C. H. Lin, L. Jiang, Y. H. Chai, H. Xiao, S .J. Chen, H. L. Tsai, Fabrication of microlens arrays in photosensitive glass by femtosecond laser direct writing, Appl Phys A 97 (2009) 751757.

[26] M.D. Perry, B.C. Stuart, P.S. Banks, M.D. Feit, V. Yanovsky, A.M. Rubenchik, Ultrashort-pulse laser machining of dielectric materials, J. Appl. Phys. 85 (1999) 6803-6810.

[27] K. Sugioka and Y. Cheng, Femtosecond laser three-dimensional micro- and nanofabrication, Applied Physics Reviews, 1 (2014) 041303-1-35.

[28] R. Wagner http://www.sciencedirect.com/science/article/pii/S0169433205016417 cor1, mailto:Ralph.Wagner@,1lt.rwth-aachen.de J. Gottmann, A. Horn, E.W. Kreutz, Subwavelength ripple formation induced by tightly focused femtosecond laser radiation, Applied Surface Science 252 (2006) 8576-8579.

[29] R. Le Harzic, F. Stracke, H. Zimmermann, Formation mechanism of femtosecond laserinduced high spatial frequency ripples on semiconductors at low fluence and high repetition rate, J. Appl. Phys. 113 (2013) 183503-183507.

[30] A. Ben-Yakar, R. L. Byer, Femtosecond laser ablation properties of borosilicate glass, J. Appl. Phys. 96 (2004) 5317-5323.

[31] A. Ben-Yakar, R. L. Byer, A. Harkin, J. Ashmore, H. A. Stone, M. Shen, E. Mazur, Morphology of femtosecond-laser-ablated borosilicate glass surfaces, Appl. Phys. Lett. 83 (2003) 3030-3032.

[32] A. Ben-Yakar, A. Harkin, J. Ashmore, R. L. Byer, H. A. Stone, Thermal and fluid processes of a thin melt zone during femtosecond laser ablation of glass: the formation of rims by single laser pulses, J. Phys. D: Appl. Phys. 40 (2007) 1447-1459. 
[33] A. Borowiec, M. Mackenzie, G. C. Weatherly, H. K. Haugen, Transmission and scanning electron microscopy studies of single femtosecond-laser-pulse ablation of silicon, Appl. Phys. A 76 (2003) 201-207.

[34] J. Yong, F. Chen, Q. Yang, G. Du , H. Bian, D. Zhang, J. Si , F. Yun , X. Hou, Rapid Fabrication of Large-Area Concave Microlens Arrays on PDMS by a Femtosecond Laser, ACS Appl. Mater. Interfaces 5 (2013) 9382-9385.

[35] Y. Ou, Q. Yang, F. Chen, Z. Deng, G. Du, J. Wang, H. Bian, J. Yong, X. Hou, Direct Fabrication of microlens arrays on PMMA with laser-induced structural modification, IEEE Photonics Technology Letters 27 (2015) 2253-2256.

[36] I. Blonskyi, V. Kadan, O. Shpotyuk, M. Iovu, P. Korenyuk, I. Dmitruk, Filament-induced self-written waveguides in glassy $\mathrm{As}_{4} \mathrm{Ge}_{30} \mathrm{~S}_{66}$, Appl Phys B 104 (2011) 951-956.

[37] P. Masselin, D. Le Coq, E. Bychkov, E. Lépine, C. Lin, L. Calvez, Laser filamentation in chalcogenide glass, Proc. of SPIE 7993 (2011) 79931B-1-6.

[38] A. Bréhault, L. Calvez, P. Adam, J. Rollin, M. Cathelinaud, B. Fan, O. MerdrignacConanec, T. Pain, X.-H. Zhang, Moldable multispectral glasses in GeS2-Ga2S3-CsCl system transparent from the visible up to the thermal infrared regions, Journal of Non-Crystalline Solids 431 (2016) 25-30.

[39] M. Born, E. Wolf, Principles of optics, Pergamon Press, 1970.

[40] C. Lin, L. Calvez, B. Bureau, Y. Ledemi, Y. Xu, H. Tao, X. Zhang, X. Zhao, Controllability study of crystallization on whole visible-transparent chalcogenide glasses of $\mathrm{GeS}_{2}-\mathrm{Ga}_{2} \mathrm{~S}_{3}-\mathrm{CsCl}$ system, Journal of Optoelectronics and Advanced Materials 12 (2010) 1684-1691.

[41] N.A. Bloembergen, A brief history of light breakdown, Journal of Nonlinear Optical Physics \& Materials 6 (1997) 377-385.

[42] C.B. Schaffer, A. Brodeur, E. Mazur, Laser-induced breakdown and damage in bulk transparent materials induced by tightly focused femtosecond laser pulses, Measurement Science and Technology 12 (2001) 1784-1797.

[43] D. von der Linde, H. Schuller, Breakdown threshold and plasma formation in femtosecond laser-solid interaction, J. Opt Soc. Am. B 13 (1996) 216-222.

[44] G. B. Airy, On the diffraction of an object-glass with circular aperture, Transactions of the Cambridge Philosophical Society 5 (1835) 283-291.

[45] I. Blonskyi, V. Kadan, O. Shpotyuk, M. Iovu, I. Pavlov, Femtosecond filamentation in chalcogenide glasses limited by two-photon absorption, Optical Materials 32 (2010) 1553-1557.

[46] K. Sokolowski-Tinten, J. Bialkowski, A. Cavalleri, D. von der Linde, A. Oparin, J. Meyerter-Vehn, S. I. Anisimov, Transient states of matter during short pulse laser ablation, Phys. Rev. Lett. 81 (1998) 224-227.

[47] D. Puerto, J. Siegel, W. Gawelda, M. Galvan-Sosa, L. Ehrentraut, J. Bonse, J. Solis, Dynamics of plasma formation, relaxation, and topography modification induced by femtosecond laser pulses in crystalline and amorphous dielectrics, J. Opt. Soc. Am. B 27 (2010) 1065-1076.

[48] T. Liu, Z. Hao, X. Gao, Z. Liu, J. Lin, Shadowgraph investigation of plasma shock wave evolution from Al target under 355-nm laser ablation, Chin. Phys. B 23 (2014) 085203-1-7.

[49] A. Miloshevsky, S. S. Harilal, G. Miloshevsky, A. Hassanein, Dynamics of plasma expansion and shockwave formation in femtosecond laser-ablated aluminum plumes in argon gas at atmospheric pressures, Physics of Plasmas 21 (2014) 043111-1-10.

[50] K. Sokolowski-Tinten, J. Bialkowski, M. Boing, A. Cavalleri, D. von der Linde, Thermal and nonthermal melting of gallium arsenide after femtosecond laser excitation, Phys. Rev. B 58 (1998) R11805-R11808.

[51] H. Kumagai, S.-H. Cho, K. Ishikawa, K. Midorikawa, M. Fujimoto, S. Aoshima, Y. Tsuchiya, Observation of the complex propagation of a femtosecond laser pulse in a dispersive transparent bulk material, J. Opt. Soc. Am. B 20 (2003) 597-602. 
[52] J. Takeda, K. Nakajima, S. Kurita, S. Tomimoto, S. Saito, T. Suemoto, Time-resolved luminescence spectroscopy by the optical Kerr-gate method applicable to ultrafast relaxation processes, Phys. Rev. B 62 (2000) 10083-10087.

[53] I. Blonskyi, M. Brodyn, V. Kadan, O. Shpotyuk, I. Dmytruk, I. Pavlov, Spatiotemporal dynamics of femtosecond filament induced plasma channel in fused silica, Appl. Phys. B. 97 (2009) 829-834. 